\title{
Spinel ferrite nanoparticles in core shell architecture for heat release
}

\author{
Marco Sanna Angotzi, ${ }^{\text {,a }}$ Valentina Mameli, ${ }^{\text {a }}$ Claudio Cara, ${ }^{a}$ Anna Musinu, ${ }^{a}$ Claudio Sangregorio, ${ }^{b}$ \\ Daniel Niznansky, ${ }^{\mathrm{c}}$ Huolin L. Xin, ${ }^{\mathrm{d}}$ Jana Vejpravova, ${ }^{\mathrm{e}}$ Carla Cannas ${ }^{\mathrm{a}}$
}

${ }^{a}$ Department of Chemical and Geological Sciences, University of Cagliari, S.S. 554 bivio per Sestu, 09042 Monserrato (CA), Italy;

${ }^{b}$ Istituto di Chimica dei Composti OrganoMetallici - Consiglio Nazionale delle Ricerche (ICCOM-CNR), Via Madonna del Piano 10, 50019 Sesto Fiorentino (FI), Italy;

${ }^{c}$ Department of Inorganic Chemistry, Charles University, Hlavova 8, 12800 Prague 2, Czech Republic; ${ }^{d}$ Center for Functional Nanomaterials, Brookhaven National Laboratory, 735 Brookhaven Ave, Upton, NY 11973, United States of America;

${ }^{e}$ Department of Condensed Matter Physics, Charles University, Ke Karlovu 5, 12116 Prague 2, Czech Republic.

$$
\text { *marcosanna@unica.com }
$$

Understanding and governing the complex behavior in magnetic materials at the nanoscale is the key and the challenge not only for fundamental research but also to exploit them in applications ranging from catalysis,[1] to data storage,[2] sorption,[3-4] biomedicine,[5-6] and environmental remediation.[7] In this context, spinel ferrites $\left(\mathrm{M}^{2+} \mathrm{Fe}^{2} \mathrm{O}^{4}\right.$, where $\mathrm{M}^{2+}=\mathrm{Fe}, \mathrm{Co}$, Mn, etc.) represent ideal magnetic materials for tuning the magnetic properties through chemical manipulations, due to their strong dependence on the cation distribution, spin-canting, interface, size, shape, and interactions. Furthermore, when coupled with other phases (heterostructures), they can display rich and novel physical properties different from the original counterparts (exchange coupling, exchange bias, giant magneto-resistance), allowing them to multiply their potential use.[8] For example, the possibility to tune magnetic anisotropy and saturation magnetization by coupling magnetically hard and soft materials have found usage recently in applications based on magnetic heat induction, such as catalysis or magnetic fluid hyperthermia (MFH).[9] Therefore, it is crucial to engineer core-shell nanoparticles with homogeneous coating and low size dispersity for uniform magnetic response and to maximize the coupling between the hard and soft phases (i.e. the interface).[10] Even though some studies have reported interesting results in the field of magnetic heat induction, a systematic study on an appropriate number of samples for a better comprehension of the phenomena to optimize the performance is needed.

In this contribution, the capability of coupled hard-soft bi-ferrimagnetic nanoparticles to improve the heating ability is exploited to understand the influence of the different features on the performances. This systematic study is then based on the correlation between the heating abilities of three magnetically hard cobalt ferrite cores, covered with magnetically soft spinel iron oxide and manganese ferrite having different thickness, with their composition, structure, morphology and magnetic properties. Direct proof of the coreshell structure formation was provided by nanoscale chemical mapping, with identical results obtained through STEM-EELS, STEMEDX, and STEM-EDX tomography. ${ }^{57} \mathrm{Fe}$ Mössbauer spectroscopy and DC/AC magnetometry proved the magnetic coupling between the hard and the soft phases, thanks also to the comparison among core-shell NPs, ad-hoc prepared mixed cobalt-manganese ferrites NPs, and cobalt ferrite NPs mechanically mixed with manganese ferrite NPs. The heating abilities of the aqueous colloidal dispersions of the three sets of core-shell samples revealed that, in all cases, core-shell nanoparticles showed better performances in comparison with the respective cores, with particular emphasis on the spinel iron oxide coated systems and the samples featuring thicker shells. This scenario entirely agrees with the hypothesis made based on magnetic parameters (saturation magnetization, Néel relaxation times, effective anisotropy) of the powdered samples, and demonstrated the importance of a sophisticated approach based on the synergy of chemical, structural, and magnetic probes down to a single-particle level. 

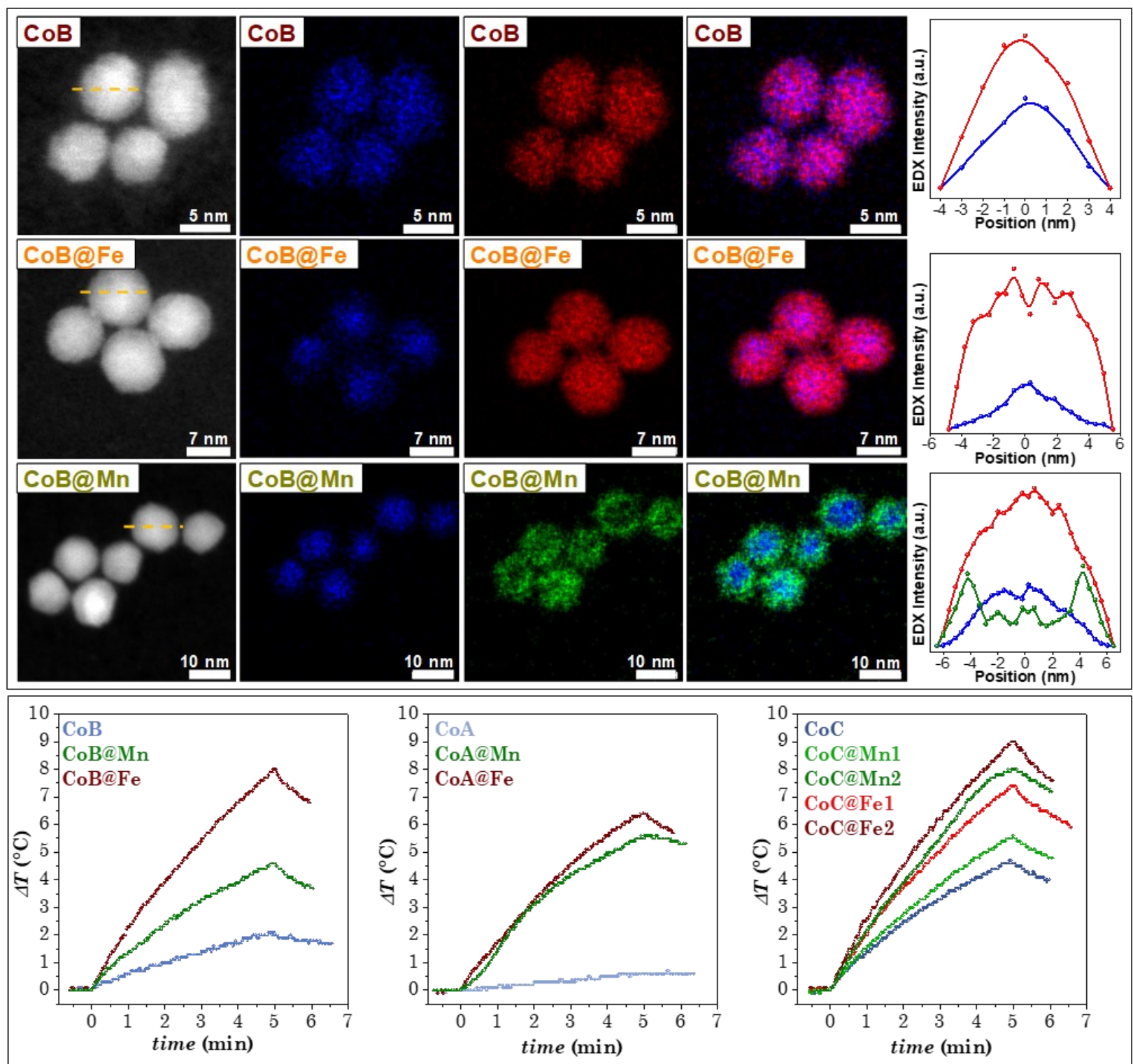

[1] Polshettiwar, V. et al. Chem. Rev., 2011, 111, 3036-3075

[2] Wu, L. et al. Nano Lett., 2014, 14, 3395-3399

[3] Cara, C. et al. J. Mater. Chem. A, 2017, 5, 21688-21698

[4] Cara, C. et al. J. Phys. Chem. C, 2018, 122, 12231-12242

[5] Lim, E. et al. Chem. Rev., 2015. 115, 327-394

[6] Mameli, V. et al. Nanoscale, 2016, 8, 10124-10137

[7] Westerhoff, P. et al. Environ. Sci. Nano, 2016, 3, 1241-1253

[8] Gawande, M.B. et al. Chem. Soc. Rev. 2015, 44, 7540-7590

[9] Lee, J.-H. et al. Nat. Nanotechnol.,2011, 6, 418-422

[10] Sanna Angotzi, M. et al. J. Nanosci. Nanotechnol., 2019, 19, 4954-4963

\section{Keywords: Spinel Ferrite Core-Shell Nanoparticles MFH}

The University of Cagliari is acknowledged for the grant financing Marco Sanna Angotzi. 